\title{
The role of oral and postingestional cues in the conditioning of taste preferences based on differing caloric density and caloric outcome in weanling and mature rats
}

\author{
LINDA HAYWARD \\ Macquarie University, North Ryde, New South Wales, Australia
}

\begin{abstract}
Experiments 1, 2, and 3 demonstrated that 23-day-old rat pups can acquire a conditioned taste preference for a flavor paired with either a more calorically dense diet or a diet that provides more calories than another diet of equal caloric density. Experiment 4 showed that 42 49-day-old rats also can acquire conditioned taste preferences based on differing caloric density and differing caloric outcome. Experiments 5 and 6 demonstrated that adult rats form conditioned taste preferences based only on caloric density. Although these experiments indicate that young rats are apparently capable of more diverse learning than adult rats, the question of what US underlies conditioned taste preferences based on diets of differing caloric density remains unresolved.
\end{abstract}

It has been well documented that rats can develop a strong aversion to a novel flavor when the initial ingestion of that flavor is followed by illness. This association can be learned even if the illness occurs some hours after presentation of the flavor (e.g., Garcia \& Koelling, 1966; Revusky \& Garcia, 1970). It has also been shown that tastes correlated with recovery from illness come to be preferred (e.g., Parker, Failor, \& Weidman, 1973; Zahorik \& Maier, 1969).

The ability of a flavor cue to become associated with cues other than illness or recovery from illness, for example, the caloric value of food, has received far less attention. Booth (1972) found evidence for the ability of flavors to elicit conditioned satiety. In his research, flavors were paired with high- and low-calorie diets during training and presented to the rats one at a time. Testing consisted of presenting the "high"- and "low"-calorie flavors one at a time in isocaloric diets. At the end of this period, a two-stimulus preference test was given. Booth found that the amount of the test meal that was consumed containing the flavor paired with the high-calorie diet was smaller than for the test meal containing the flavor paired with the lowcalorie diet. However, in a two-stimulus preference test, the flavor paired with the high-calorie diet was preferred. Holman (1975) showed that taste preferences can be conditioned on the basis of oral con-

I would like to thank R. F. Westbrook, N. W. Bond, and W. D. Hall for their suggestions and comments on drafts of this paper. My mailing address is: School of Behavioural Sciences, Macquarie University, North Ryde, N.S.W. 2113, Australia. sequences alone, as well as on the basis of postingestional effects. In his experiments, a flavor followed immediately by a nonnutritive relatively sweet taste (saccharin) came to be preferred, whereas if the presentation of the nonnutritive sweet taste was delayed, no preference was observed. However, if a flavor was followed by a nutritive sweet taste (dextrose), a preference was observed with a delay.

Bolles, Hayward, and Crandall (1981) investigated the relative importance of oral cues and postingestional consequences for flavor preference learning. During training, flavors were added to diets of high and low caloric density presented simultaneously. On tests employing a midcaloric density diet, the flavor previously paired with the highcalorie diet was preferred. A preference for the highcalorie diet itself was observed very early in the training period, and this, along with the observation that, on the initial presentation, rats sample first one and then the other diet in quick succession, seemed to indicate that the US operating in these experiments was an oral cue of some sort. The final experiment in that paper compared two flavors paired with diets of equal caloric density but different caloric outcome in one group, and two flavors paired with diets of differing caloric density but equal caloric outcome in another group. They found no preference for flavors paired with diets of differing caloric outcome, but did find a preference for flavors paired with a diet of high caloric density. Bolles et al. concluded that there was no indication that postingestional consequences had anything to do with the conditioning of taste preferences in their experiments. Evidently, an oral cue 
of some sort was functioning as a US, and perhaps they were looking at a different aspect of intake regulation from earlier workers, for example, food selection rather than the regulation of food consumption over time.

The question of what mechanism would underlie an oral US remains unresolved. There are many possibilities; it could be that the rat innately prefers starchy food, or that the rat has to learn about the properties of starch. Perhaps early in life the rat learns to associate postingestional consequences with the oral properties of food like starch, eventually being able to rely entirely on oral cues, since being able to sample food and immediately ascertain its worth would make foraging more efficient. The experiments in this paper examine the development of taste preference learning in rat pups on the premise that the behavior of rat pups will shed some light on the issue.

\section{EXPERIMENT 1}

The design of this experiment is the same as that used by Bolles et al. (1981) in their final experiment. The purpose of the following experiment is to see whether rat pups can acquire a conditioned flavor preference based on caloric outcome as well as caloric density. When Bolles et al. did the experiment with adult rats, they found that flavor preferences were formed on the basis of caloric density but not caloric outcome. If rat pups pay attention to postingestional feedback, then one might expect to find preferences based on differing caloric outcome as well as differing caloric densities.

\section{Method}

Subjects. Sixteen Wistar rat pups were used. They were 23 days old at the start of the experiment and had just been weaned.

Materials. The high-calorie food consisted of $50 \%$ dextrin, $15 \% \mathrm{CaCO}_{3}, 2.5 \%$ mineral oil, $7.5 \%$ vegetable oil, and $25 \%$ lactic casein. It provided approximately $4 \mathrm{cal} / \mathrm{g}$. The low-calorie food consisted of $15 \%$ dextrin, $50 \% \mathrm{CaCO}_{3}, 7.5 \%$ mineral oil, $2.5 \%$ vegetable oil, and $25 \%$ lactic casein. It provided approximately $2 \mathrm{cal} / \mathrm{g}$. Either anise or coconut extract was added to the diets.

Procedure. The rats were assigned randomly to two groups. Group 1 (calorie group) received one flavor in $4 \mathrm{~g}$ of the 4-cal/g diet and the other flavor in $2 \mathrm{~g}$ of the $4-\mathrm{cal} / \mathrm{g}$ diet on alternate days. For these rats, the two flavors were correlated with the same pattern of oral cues and the same caloric density but different caloric consequences ( 16 vs. 8 calories).

Group 2 (oral group) were given one flavor in $3 \mathrm{~g}$ of the 4$\mathrm{cal} / \mathrm{g}$ diet and the other flavor in $6 \mathrm{~g}$ of the 2-cal/g diet on alternate days. For these rats, the two flavors were correlated with the same caloric consequences (12 vs. 12 calories) but different caloric densities and oral cues.

Lab chow was removed $2.5 \mathrm{~h}$ prior to the presentation of the diet and returned $5 \mathrm{~h}$ after presentation, and usually in this time all the food had been eaten. Flavors and the positions of food jars were counterbalanced. Conditioning continued for 8 days, 4 days with each diet. A test with the flavors added to two jars of 3-cal/g food was undertaken on the 9th day. The test diets were presented simultaneously. The amount consumed was recorded after $30 \mathrm{~min}$.

Because one of the rats in Group 2 died during training, the analysis of the data is based on eight rats in Group 1 and seven rats in Group 2.

\section{Results and Discussion}

The data were analyzed using a repeated-measures analysis of variance. There was no main effect for groups $(F<1)$, indicating that there was no difference between the two groups in the total amount consumed on the test day. There was a main effect for the type of diet $[F(1,13)=6.35, p<.05]$; that is, when averaged across groups, the flavor paired with either the more calorically dense food or the food that provided more calories was preferred (see Figure 1). There was no interaction between group and type of diet $(F<1)$.

These data indicate that 23-day-old rat pups are capable of acquiring a conditioned taste preference based either on caloric density or on caloric outcome. This finding is of interest in that young rats seem to be capable of more diverse learning than adults. It also opens up the possibility that young rat pups might use postingestional feedback when forming associations between flavors paired with foods of differing caloric density as well as differing caloric outcome.

The reason for the death of the rat pup in Group 2 is unknown. Since it was possible that the pups were sick and the data are thus not representative, the first experiment was replicated.

\section{EXPERIMENT 2}

\section{Method}

Subjects. Sixteen 23-day-old Wistar rat pups were used. They were weaned on Day 1 of the experiment.

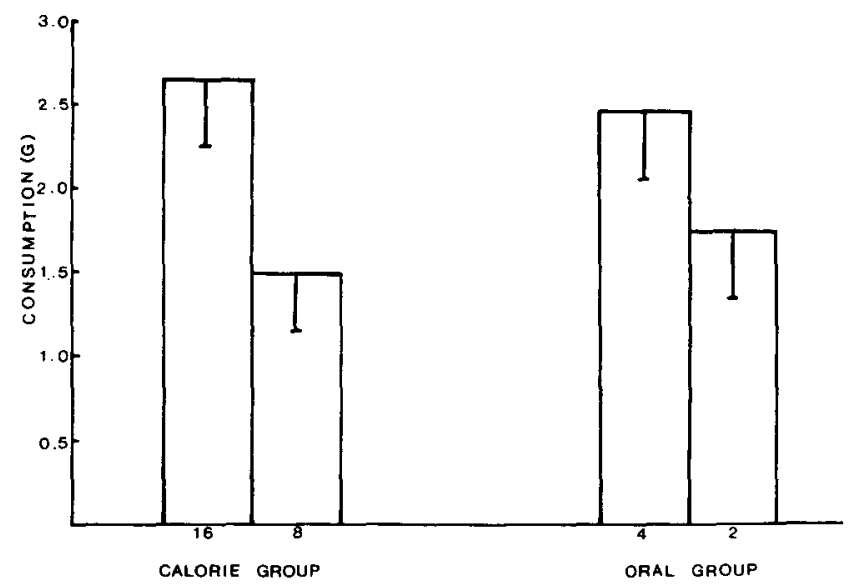

Figare 1. Mean consumption of the two flavors on test day. For the calorie group, one flavor had been paired with $16 \mathrm{cal}$ of the $4-\mathrm{eal} / \mathrm{g}$ diet, the other with $8 \mathrm{cal}$ of the $4-\mathrm{cal} / \mathrm{g}$ diet. For the oral group, one flavor had been paired with 12 cal of the $4-\mathrm{cal} / \mathrm{g}$ diet and the other with $12 \mathrm{cal}$ of the $2-\mathrm{cal} / \mathrm{g}$ diet. 
Materials and Procedure. The materials and procedure were the same as for Experiment 1.

\section{Results and Discussion}

The data were analyzed using a repeated-measures analysis of variance. There was no main effect for groups $[F(1,14)=2.53]$, in the amount of food consumed on the test day. There was a main effect for days and for the type of diet $[F(1,14)=9.07$, $\mathrm{p}<.01]$, indicating that, when averaged across groups, there was a preference for the flavor paired with either the more calorically dense diet or the food that provided more calories. The interaction was also significant $[F(1,14)=7.97, p<.05]$. From Figure 2, it appears that there was no preference in the calorie group, while there was one in the oral group. However, the data showed that six of the rats in the calorie group did show a preference for the flavor that had been paired with the food providing more calories, the other two showing a markedly strong preference in the opposite direction.

Perhaps the conditioning in the calorie group was weaker, or more variable, because of something to do with the test food. For animals in the oral group, the test diet $(3 \mathrm{cal} / \mathrm{g})$ lay midway between the densities of the training diets $(4$ and $2 \mathrm{cal} / \mathrm{g}$ ); for the calorie group, the test diet $(3 \mathrm{cal} / \mathrm{g}$ ) was less dense than either of the training diets (both $4 \mathrm{cal} / \mathrm{g}$ ). It is possible that conditioning is in some way interfered with for rats in the calorie group, when faced with a novel, less palatable diet than they are accustomed to. Another problem with this design, especially with adult rats, is that a flavor that is paired with more or less of a food that accounts for only a small proportion of their daily caloric intake may not be attended to. For these

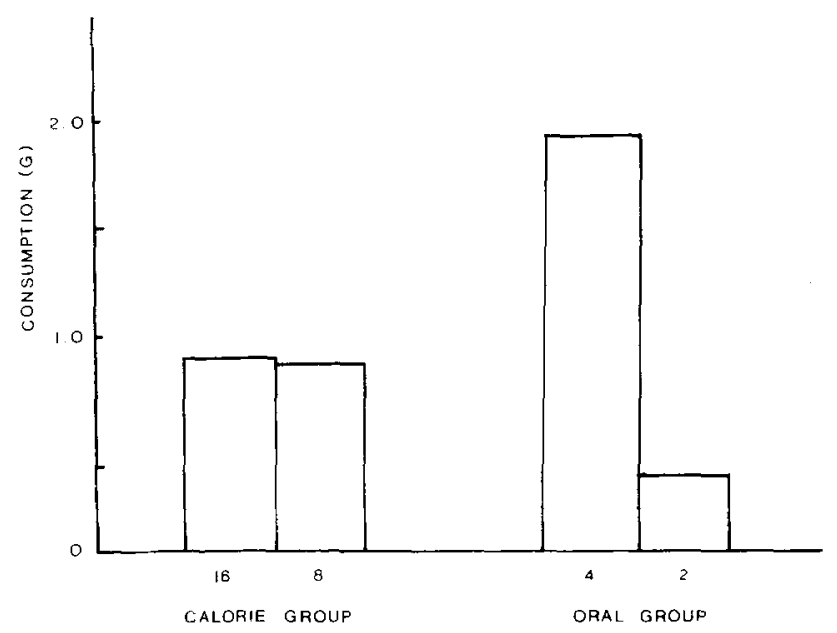

Figure 2. Mean consumption of the two navors on test day. For the calorie group, one flavor had been paired with $16 \mathrm{cal}$ of the $4-\mathrm{cal} / \mathrm{g}$ diet, the other with $8 \mathrm{cal}$ of the $4-\mathrm{cal} / \mathrm{g}$ diet. For the oral group, one flavor had been paired with $12 \mathrm{cal}$ of the $4-\mathrm{cal} / \mathrm{g}$ diet and the other with $12 \mathrm{cal}$ of the $4-\mathrm{cal} / \mathrm{g}$ diet. reasons, the following experiment used rat pups, but the design was changed to see whether conditioning could be improved when the diets formed the entire daily food intake and when the test food was $4 \mathrm{cal} / \mathrm{g}$.

\section{EXPERIMENT 3}

Since it had been established in the first two experiments that rat pups could acquire a strong preference for a flavor that had previously been paired with a calorically dense food, the present experiment concentrated solely on conditioning based on caloric outcome. The main change from the calorie groups in the earlier experiments was that this time the test food was $4 \mathrm{cal} / \mathrm{g}$ (the same as the training diets), and the diets represented the major part of the animal's daily caloric intake. The latter change is not so important for rat pups because the quantities given in the earlier design did account for a large part of their daily needs. However, it does become important for adult rats, since these quantities represent less than a quarter of their daily caloric requirements. In this experiment, rats were given unlimited access to the diet on 1 day, and three-quarters of the previous day's consumption on the next day. Since it was possible that the flavors would be associated with the presence or absence of hunger, rather than with the monitoring of actual caloric intake, the rats were divided into two groups: one group underwent the procedure described above, and the other group received a supplement of lab chow on Days 2 and 4 when they received three-quarter rations, thus ensuring that this group not be differentially hungry on these days.

\section{Method}

Subjects. Sixteen 23-day-old Wistar rat pups were used. They were weaned on Day 1 of the experiment.

Materials. The 4-cal $/ \mathrm{g}$ diet and the flavors were the same as in Experiment 3.

Procedure. On conditioning days, the rat pups were removed from their mothers and housed individually with ad-lib access to water. The rats were assigned randomly to two groups. Group 1 received one flavor in $20 \mathrm{~g}$ of the $4 \mathrm{cal} / \mathrm{g}$ on Day 1 . They had access to this food for $24 \mathrm{~h}$, at the end of which consumption was measured. On Day 2, each rat received three-quarters of its previous day's intake, and again the diet used was $4 \mathrm{cal} / \mathrm{g}$, but this time the other flavor was added. They had access to this diet for $24 \mathrm{~h}$, but usually consumed it within $8 \mathrm{~h}$, so that they went hungry for the latter part of the $24-\mathrm{h}$ period. At the end of the 24-h period, the procedure for Days 1 and 2 was repeated. Altogether, the rats in this group experienced 2 days with sufficient food and 2 days with an inadequate amount of food. Group 2 underwent the same procedure and had the same amount of training as Group 1, but on Days 2 and 4 the food supply for these animals was topped up with lab chow $8 \mathrm{~h}$ after presentation of the diet.

Both groups were given a 30 -min test on Day 5. Two jars of 4-cal $/ \mathrm{g}$ food were presented simultaneously, and consumption was recorded at the end of the 30 -min period. Flavors and position of jars were counterbalanced throughout the experiment. 


\section{Results and Discussion}

On Day 1 an average of 5.35 and $4.52 \mathrm{~g}$ and on Day 2 an average of 3.91 and $3.8 \mathrm{~g}$ of food were eaten by Groups 1 and 2, respectively. Test-day consumption was analyzed using a repeated-measures analysis of variance. There was no main effect for groups $(F<1)$ or a group $\times$ calorie interaction $(F<1)$. There was a main effect for caloric outcome $[F(1,14)=16.45, p<.01]$, indicating that more of the food containing the flavor paired with more calories was eaten (see Figure 3).

This experiment clearly demonstrates that rat pups are capable of forming a conditioned flavor preference based on caloric intake. Moreover, this preference was stronger than that shown by the calorie groups in the previous two experiments in that on test day hardly any of the food containing the flavor paired with lower caloric outcome was consumed. One reason for this stronger preference might be that the rats were required to carry out a more meaningful task. This time they were required to learn to associate a flavor with a food that had consistently been more plentiful in the past. It was not necessary to use a different test stimulus, since, unlike rats given foods of different density, there were no cues other than the added flavor to influence choice in a short test.

It is possible that the present findings differ from those of Bolles et al.(1981) because of slight procedural differences and changes to the components of the test diets; for example, the present diets contained some protein in the form of lactic casein.

The next experiment was carried out to rule out

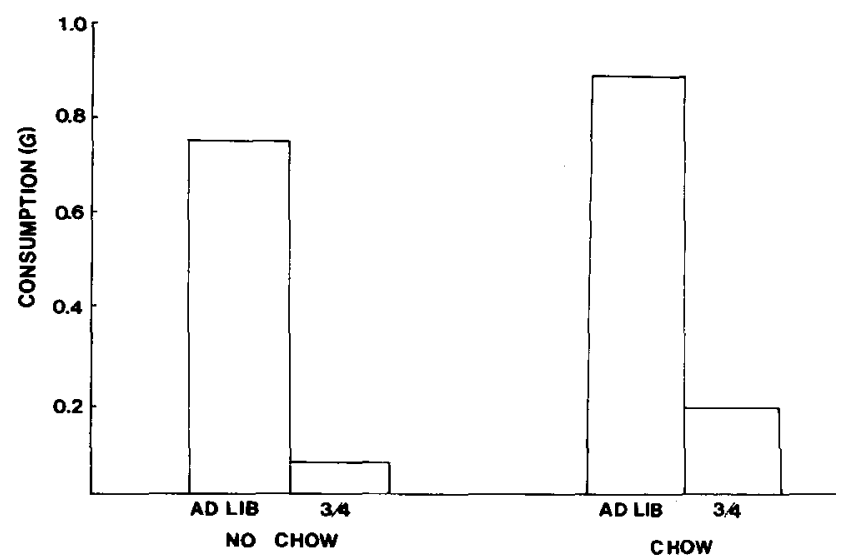

Figure 3. Mean consumption of the two fiavors on test day. For the no-chow group, the two flavors were paired with $4-c a l / g$ food, but one fiavor had been paired with ad-lib access to $4-$ cal/g food on one day and the other flavor had been paired with three-quarters of the amount of food consumed on ad-lib access the following day. For the chow group, flavors were paired with diets in the same way as for the no-chow group, except that the day on which three-quarter rations were presented these animals had access to lab chow. this possibility. If the findings were not due to procedural differences, then a preference was predicted in the oral group but not the calorie group. This experiment followed the design of Experiment 1, in order to provide a replication of the Bolles et al. study.

\section{EXPERIMENT 4}

\section{Method}

Subjects and Materials. Sixteen Wistar rats were used. They were 42-49 days old on test day. They had had 21-28 days' experience with solid food in the form of lab chow. The high- and lowcalorie diets and added flavors were the same as those used in Experiments 1 and 2.

Procedure. The procedure was the same as for Experiments 1 and 2, except that the amount of food presented to the two groups was the same as that presented by Bolles et al. (1981). The rats were assigned randomly to two groups. Group 1 (calorie group) received one flavor in $3 \mathrm{~g}$ of the $4-\mathrm{cal} / \mathrm{g}$ diet and the other flavor in $1.5 \mathrm{~g}$ of the 4-cal/g diet on alternate days.

Group 2 (oral group) were given one flavor in $1.5 \mathrm{~g}$ of the $4-\mathrm{cal} / \mathrm{g}$ diet and the other flavor in $3 \mathrm{~g}$ of the $2-\mathrm{cal} / \mathrm{g}$ diet on alternate days. Also, only one test was carried out after the 8 conditioning days, and a record of the amount consumed was taken after $30 \mathrm{~min}$ and $3 \mathrm{~h}$.

\section{Results and Discussion}

The mean consumptions for both groups at $30 \mathrm{~min}$ and $3 \mathrm{~h}$ on test day are shown in Figure 4. There was no difference between groups in total food consumption when averaged across time of testing or food value $(F<1)$. The flavor paired with either the more calorically dense food or the food that provided more calories was preferred when averaged across groups and time of testing $[F(1,14)=42.96, p<.01]$. The difference between groups in the preference for rich food or food providing more calories when averaged across time did not reach significance $[F(1,14)=3.97]$. However, from Figure 4 , it can be seen that rats in the calorie group had a tendency to eat more of the food containing the nonpreferred flavor than did rats in the oral group, indicating that conditioning in the oral group is stronger.

Taste preferences were found in both groups. The calorie group preferred a flavor paired with high caloric outcome, and the oral group preferred a flavor paired with high caloric density, but the preference in the oral group appeared to be stronger. The preferences held across both the $30-\mathrm{min}$ and 3-h tests.

This experiment demonstrated that, at $42-49$ days of age, rats use postingestional consequences as a basis for a taste preference. This result was not as predicted, although it is possible that, at this age, lack of experience with starchy foods and immaturity of the neurological systems involved in ingestion control (Kolb \& Nonnemann, 1976, found that maturation of these systems continues even after 60 days of age) were responsible for these 


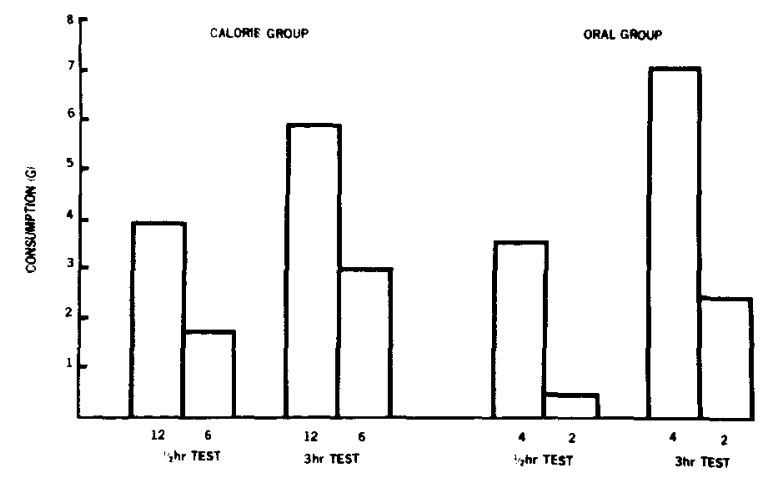

Figure 4. Mean consumption of the two flavors at 30 -min and 3-h tests. For the calorie group, one flavor had been paired with $12 \mathrm{cal}$ of the 4-cal $/ \mathrm{g}$ diet, the other with $6 \mathrm{cal}$ of the $4-\mathrm{cal} / \mathrm{g}$ diet. For the oral group, one flavor had been paired with $6 \mathrm{cal}$ of the $2-\mathrm{cal} / \mathrm{g}$ diet, and the other with $6 \mathrm{cal}$ of the $4-\mathrm{cal} / \mathrm{g}$ diet.

results' being similar to those obtained with weanling rat pups.

The next experiment was an attempt to replicate Bolles et al., using mature rats.

\section{EXPERIMENT 5}

\section{Method}

Subjects. Sixteen 5- to 6-month-old Wistar rats were used.

Materials and Procedure. The materials and procedure were the same as for Experiment 4.

\section{Results and Discussion}

The mean consumptions for both groups at $30 \mathrm{~min}$ and $3 \mathrm{~h}$ on test day are shown in Figure 5. On test day, there was no difference in the total amount of food consumed by either group when averaged across time $[\mathrm{F}(1,14)=2.4]$. However, there was a difference between the groups in the preference for the flavor paired with either the more calorically dense diet or the food that provided more calories $[F(1,14)=12.63, p<.01]$. From Figure 5, it can be seen that rats in the oral group developed a preference for a flavor paired with a more dense food,

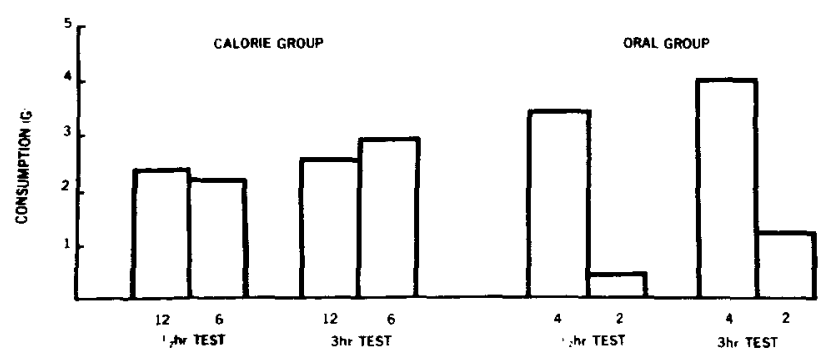

Figure 5. Mean consumption of the two navors at 30-min and 3-h test. For the calorie group, one finvor had been paired with $12 \mathrm{cal}$ of the $4 \mathrm{cal} / \mathrm{g}$ diet, and the other with $6 \mathrm{cal}$ of the $4-\mathrm{cal} / \mathrm{g}$ diet. For the oral group, one flavor had been paired with $6 \mathrm{cal}$ of the 2-cal $/ \mathrm{g}$ diet, and the other with $6 \mathrm{cal}$ of the $4-\mathrm{cal} / \mathrm{g}$ diet. but that rats in the calorie group showed no flavor preferences. This experiment provided a replication of the final study in the Bolles et al. (1981) paper and eliminated the possibility of procedural differences.

\section{EXPERIMENT 6}

This experiment was a replication of Experiment 3, this time using adult rats. It is possible that one reason why conditioning was not observed in Experiment 5 in the calorie group is that the amount of food presented to them during training made up only a small proportion of their daily requirements, so that the flavors were not attended to. Another reason why conditioning was not observed in the calorie group in Experiment 5 might be that an adult rat no longer attends to postingestional feedback, since it is rarely hungry. Perhaps the only relevant information for a laboratory rat is how calorically dense the food is, so that the most value for the least effort can be obtained. The following experiment attempted to see whether adult rats could be responsive to flavors paired with differing caloric outcomes when the experimental diets provide most of the rats' daily caloric needs.

\section{Method}

Subjects. Sixteen 65-70-day-old Wistar rats were used.

Materials and Procedare. The materials and procedure were the same as for Experiment 3 except that on test day consumption was measured after $30 \mathrm{~min}$ and $3 \mathrm{~h}$.

\section{Results and Discussion}

The following results are based on eight rats in Group 1 and seven rats in Group 2, since one rat in Group 2 would not consume the diet on Day 1. The mean consumption for both groups after $3 \mathrm{~h}$ on test day is shown in Figure 6. Test day consumption was analyzed using a repeated-measures analysis of variance. The only contrast that achieved significance was one that compared the total consumption averaged across groups at $30 \mathrm{~min}$ and $3 \mathrm{~h}$ $[F(1,13)=4.8, p<.05]$. From Figure 6, it can be seen that more is consumed at $3 \mathrm{~h}$ than at $30 \mathrm{~min}$, which is hardly surprising. There was no indication of any preference for the flavor paired with the food that provided more calories $(F<1)$. It appears that even when the diets provide most of the rats' daily caloric needs, adult rats do not learn to associate a flavor with a diet that provides more calories. This finding supports that of Bolles et al. (1981).

\section{GENERAL DISCUSSION}

Experiments 1 and 2 demonstrated that 23-dayold rat pups can acquire a conditioned taste preference for a flavor paired with either a more calorically dense diet or a diet that provides more calories 


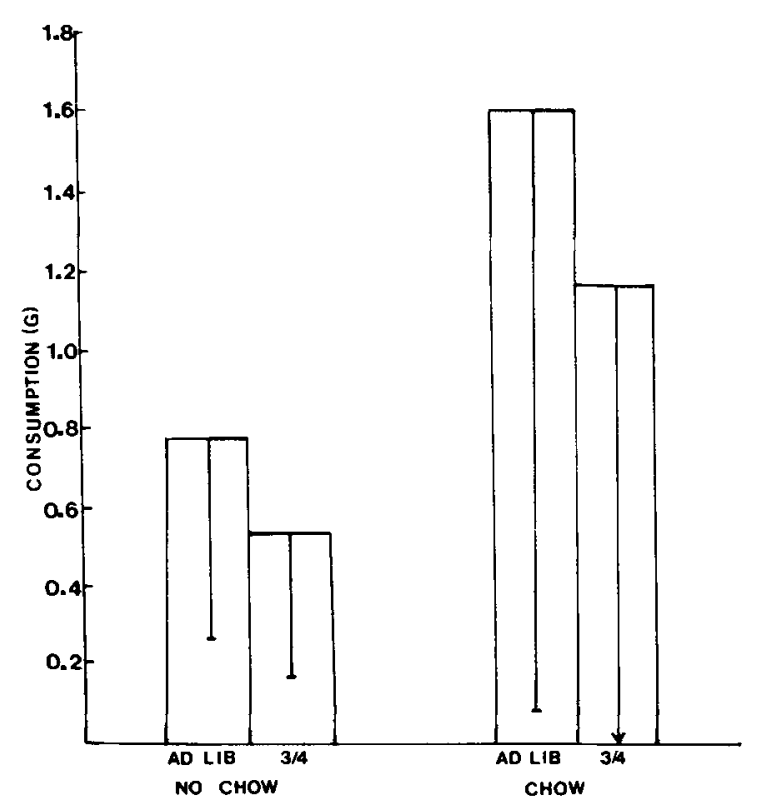

Figure 6. Mean consumption of the two navors at 3 h on test day. For the no-chow group, the two navors were paired with $4-c a l / g$ food, but one flavor had been paired with ad-lib access to the food on one day and the other flavor had been paired with three-quarters of the amount of food consumed on ad-lib access on the following day. For the chow group, flavors were paired with diets in the same way as in the no-chow group, except that the day on which three-quarter rations were presented these animals had access to lab chow.

than another diet of equal density. However, in Experiment 2, conditioning in the calorie group was far weaker than in the oral group, with only six of the eight rats showing a preference for the flavor paired with the food that provided more calories. An attempt was made in Experiment 3 to demonstrate stronger conditioning in a calorie group by altering the experimental design.

In Experiment 3, rat pups were given foods of equal caloric density but different caloric outcome on alternate days, and testing was carried out using a food of the same caloric density as that used in training. Using this design, strong conditioning to the flavor paired with the food providing more calories was demonstrated; that is, results from this experiment more closely resembled the pattern observed in oral groups, which consumed very little of the food containing the nonpreferred flavor. Experiment 4 showed that 42-49-day-old rats can acquire conditioned taste preferences to flavors paired with diets of high caloric density or greater caloric outcome. These results differ from those obtained from more mature rats in Experiments 5 and 6 . Mature rats acquired a conditioned taste preference only to flavors paired with diets of high caloric density.

Taken together, these findings indicate that young rats can use postingestional cues and oral cues as the basis for acquiring a flavor preference, whereas mature rats appear to be ignoring postingestional cues and relying solely on oral cues.

There are at least two ways of explaining this pattern of results. One is to consider them in a developmental framework. Rat pups, up until at least 10 days of age, do not respond to postingestional cues other than distension. Hall and Rosenblatt (1978) found that in 10-day-old pups both nutritive and nonnutritive stomach preloads depressed diet intake during suckling by the same amount, apparently as a result of gastric distension, whereas in 20-day-old pups diet intake was differentially depressed only by nutritive preloads. They suggested that these results indicate the emergence of postabsorptive or postgastric control of behavior. Therefore, by 20 days of age (just prior to weaning), rat pups have started attending to postingestional feedback that will allow them to forage efficiently once weaned. However, it would be inefficient to rely on postingestional feedback alone in selecting food, because of the time lapse between ingestion and receiving information about the food value. If, instead, the rat learned to associate postingestional feedback with the oral properties of food (e.g., texture and taste), selection would be far more efficient, since the animal would know the value of the food as soon as it was in its mouth. Booth (1979) proposed that this could be achieved by classical conditioning, such that the oral properties of food and the animals' bodily state were CSs present during a meal and that these were conditioned by metabolic USs that continue after a meal.

In the present series of experiments, it is conceivable that the mature rats were not attending to postingestional feedback because of long experience with a relatively starchy food (lab chow); that is, they may have already learned about the oral properties of starch. When faced with the task of discriminating between diets, they simply have to associate the added flavor with the oral properties of the diet. Young rats, however, have not had much experience with starchy foods and therefore are still attending to postingestional cues as well as what they may have already learned about the oral properties of starch.

There is another way of looking at the present results. The fact that rat pups can learn to associate a flavor with a food that provides more calories does not rule out the possibility that they innately prefer starchier food. The experimental design used in Experiments 1 and 2, although allowing us to ask whether rat pups can use postingestional feedback as a basis for a flavor preference, do not allow us to assume that such a mechanism is being used when they show a flavor preference for a flavor paired with a calorically dense food. It may well be that rats do have an innate preference for starchy 
foods and that they use postingestional mechanisms to learn about food as well. Perhaps both systems are utilized by the young rat, whereas the adult rat tends to rely more heavily on oral cues. The fact that there is some evidence for flexibility when oral cues are used does not make the adult rats' strategy seem as precarious as it sounds. Bolles et al., for instance, noted that if, after training, the flavors paired with rich and poor were reversed, the rats appropriately switched their preference, and yet, when the flavors were added to novel isocaloric foods, the rich-paired flavor was tracked for a relatively long time. London, Snowdon, \& Smithana (1979) have shown that rat pups come to prefer innately aversive flavors (sour and bitter) if they are paired with food during critical periods, either during nursing or in the early postweaning period.

The present findings indicate: (1) that young rats are capable of using postingestional feedback as a basis for acquiring a conditioned taste preference for a flavor paired with a diet that provides greater caloric outcome, whereas adult rats are not; (2) that both adult and young rats are capable of forming a conditioned taste preference for a flavor paired with a diet of greater caloric density. However, it has not been established what the US might be in this case. For adult rats, who very quickly establish a preference during training for the more calorically dense food, it appears that the US is oral. For rat pups, the answer is still unclear. It may be that rat pups have an innate preference for starchy foods, or that they need to learn about the oral cues that go along with starchiness.

\section{REFERENCES}

Bolles, R. C., Hayward, L., \& Crandall, C. Conditioned taste preferences based on caloric density. Journal of Experimental Psychology: Animal Behavior Processes, 1981, 7, 59-69.

Booth, D. A. Conditioned satiety in the rat. Journal of Comparative and Physiological Psychology, 1972, 81, 457-471.

Booth, D. A. Metabolism and the control of feeding in man and animals. In K. Brown \& S. C. Cooper (Eds.), Chemical influences on behaviour. London: Academic Press, 1979.

Garcia, J., \& Koeldina, R. A. Relation of cue to consequence in avoidance learning. Psychonomic Science, 1966, 4, 123-124.

Hall, W. G., \& RosenblatT, J. S. Development of nutritional control of food intake in suckling rat pups. Behavioral Biology, $1978,24,413-427$.

Holman, E. W. Immediate and delayed reinforcers for flavor preferences in rats. Learning and Motivation, 1975, 6, 91-100.

Kole, B., \& Nonneman, A. J. Functional development of prefrontal cortex in rats continues into adolescence. Science, 1976, $193,335-336$.

London, R. M., Snowdon, C. T., \& Smithana, J. M. Early experience with sour and bitter solutions increases subsequent ingestion. Physiology \& Behavior, 1979, 22, 1149-1155.

Parker, L., Failor, A., \& Weidman, $K$. Conditioned preferences in the rat with an unnatural need state: Morphine withdrawal. Journal of Comparative and Physiological Psychology, 1973, 82, 294-300.

Revusky, S. H., \& Garcia, J. Learned associates over long delays. In G. H. Bower \& J. T. Spence (Eds.), Psychology of learning and motivation: Advances in research and theory (Vol. 4). New York: Academic Press, 1970.

Zahorik, D. M., \& MAler, S. F. Appetitive conditioning with recovery from thiamine deficiency as the unconditioned stimulus. Psychonomic Science, 1969, 17, 309-310.

(Manuscript received August 10, 1982; revision accepted for publication March 17, 1983.) 